\title{
Daño neurológico en el niño II. (Aspectos acerca de la magnitud - asistencia - profilaxis y expectativas)
}

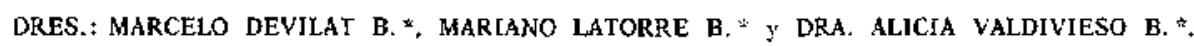

\section{MAGNITUD DEL PROBLEMA:}

Es muy difícil determinar en forma exacta la magnitud del Daño Neurológico en el niño en las categorías de cuadros clínicos ya reseñados en la primera parte de este trabajo. Los autores extranjeros dan cifras que no se corresponden entre sí y en su mayoría son "apreciaciones" para los distintos países basados en estudios efectuados con metodologías diferentes (1) (2) (3) (4).

En Chile, no hay estadísticas definitivas sobre este grupo de enfermedades, por cuanto no son habitualmente un diagnóstico de defunción, no son enfermedades denunciables $y$ sobre todo algunas de ellas se diagnostican con escasa frecuencia (5). Por otra parte no conocemos estudios estadísticos específicos que sobre estos problemas hayan sido realizados en nuestro país, salvo uno realizado en 1966 que toca algunos de los temas aquí analizados (6) (7).

En base a lo anterior mostrareros la magnitud del problema en Chile de acuerdo a datos obtenidos de otros países, suponiendo que la atención nacional del embarazo, parto, recién nacido e infantil es similar a la extranjera. Trabajaremos con una población general estimada de 10.000 .000 de habitantes (1970: 9.726.000; 1971: 9.922.000) (8) y una tasa de natalidad de $25,1 \%$ (9), calculamos la población infantil como el $36 \%$ de la población general (10).

En la tabla No 1 se presenta la frecuencia de la P. C. en Chile, dos autores, Paine (3) y Lindemann, (2), ocupando el mismo patrón de análisis: población infantil, dan cifras tan disímiles como 18.000 y 68.000 paralíticos cerebrales entre los niños de Chile. Ahora, si nos atenemos at Altman (2) habrían 40.000 enfermos con Pará-

\footnotetext{
*Departamento de Neurología, Hospital "Luis Calvo Mackenna".
}

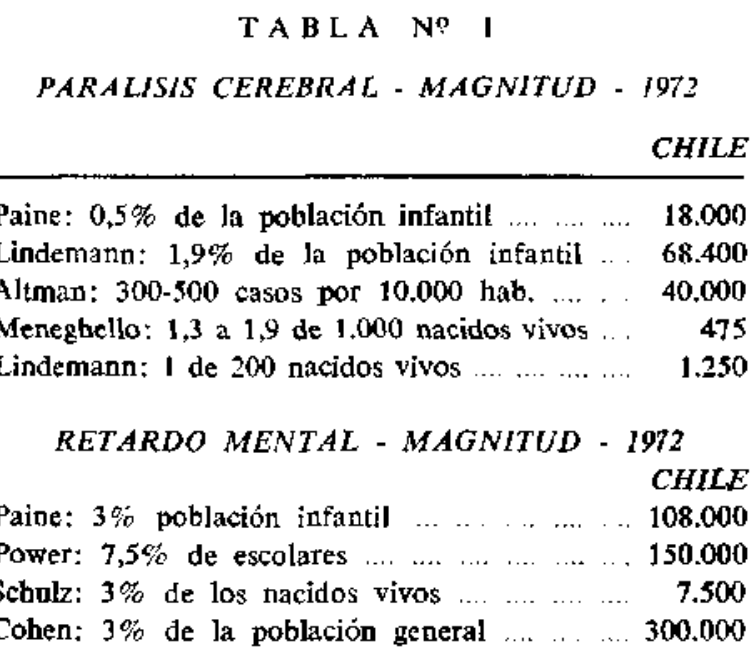

lisis Cerebral en toda la población. Variaciones tan importantes como las anteriores encontramos si analizamos la aparición de la P. C. por nacióos vivos.

En la misma tabla vemos que según Paine (3) habrían $108.000 \mathrm{R}$. M. entre la población infantil. Power, (7) en Chile, en una muestra representativa de todos los niveles económicos, compuesta por 1.894 escolares da para este solo grupo de niños 150.000. Por su parte, Cohen, (11) da un porcentaje bastante más alto, de tal manera que habrían en nuestro país $300.000 \mathrm{R}$. M.

En la tabla No 2, se muestran las cifras de Epilepsia. La misma situación anterior se observa aquí. Paine (3) por ejemplo daría 18.000 epilépticos entre todos los niños de Chile. Power (7) en cambio comunica que sólo entre los escolares habrían 44.000. Ford (12) y Meneghello (4) dific- 
TA B L A N 2

EPILEPSIA - MAGNITUD - 1972

CHILE

Paine: $0,5 \%$ de la población infantil

18.000

Power: $2,2 \%$ de los escolares

44,000

Ford: $3 \%$ de la población general .... ...... 330.000

Meneghello: $0,5 \%$ de lá población general ... 50.000

Crowther: $6 \%$ de la población general 1 crisis 600.000

Meneghello: 2 a $3 \%$ de la población infantil

I crisis

108.000

DISFUNCION CEREBRAL MINIMA - MAGNITUD 1972

Paine: $5 \%$ de la población infantil

180.000

TABLA No 3

ALTERACIONES SENSO PERCEPTIVAS

MAGNITUD - 1972

CHILE

\section{OCULARES}

Estrabismo:

Lindemann: 3,5\% Población general

350.000

Meneghello: 5\% Población escolar .... ... 100.000

Power: $17,6 \%$ Población escolar

352.400

Ceguera:

Wallin: 2\% Población escolar

Wallin: 1,5\% Población general

3.000

\section{AUDITIVAS}

Hipoacusia:

Power: $4,6 \%$ Población escolar

92.000

Sordera:

Power: $0,3 \%$ Población escolar

\section{DISLEXIA CONGENITA ESPECIFICA}

Critchey: < 10\% Población escolar

200.000

Paine: 3\% Población escolar .... ...

60.000

Brown: $10 \%$ Población escolar

200.000

Meneghello: 15\% Población escolar
350.000 y 92.000 respectivamente. Con respecto a la dislexia congénita específica, también es un problema de envergadura y dos de los cuatro autores citados dan una frecuencia similar de 200.000 escolares.

Las cifras presentadas revelan aproximadamente por cierto, que entre 500.000 a 800.000 niños padecen alguna de las enfermedades que aquí comentamos. Pensemos que ellas pueden ser rebajadas en la medida que mejoren las prestaciones del equipo médico a la embarazada, recién nacido y población infantil. Inversamente sin embargo, las acciones de salud a ese nivel dejan sobrevivientes, muchos de ellos con secuelas neurológicas, que antes fallecían y ahora es posible que incrementen el número de niños con daños cerebrales.

Desde el punto de vista asistencial, destacamos que en el Servicio de Psiquiatría Infantil del Hospital Luis Calvo Mackenna (HCM) las más frecuentes causas de consultas, corresponden a DCM, Epilepsia, RM. y problemas de aprendizaje (13). El $30 \%$ de las consultas a Sicología de ese mismo Hospital corresponden a DCM (14). El 4\% de las consultas de los Servicios de Urgencia pediátricos de los Hospitales Arriarán y Roberto del Río son por convulsiones (4) y en los Servicios de Neurologia Infantil, la Epilepsia es la causa más frecuente de consulta.

\section{DERIV ACION DE LOS CASOS - RECUR- SOS Y POSIBILIDADES DE ASISTENCIA:}

En la figura No 1 se observan los distintos niveles de recursos que existen en el país para enfrentar los problemas de los niños con Daño Neurológico. Un primer nivel lo constituyen las instituciones o profesionales que deben sospechar, generalmente en primer término, las alteraciones de

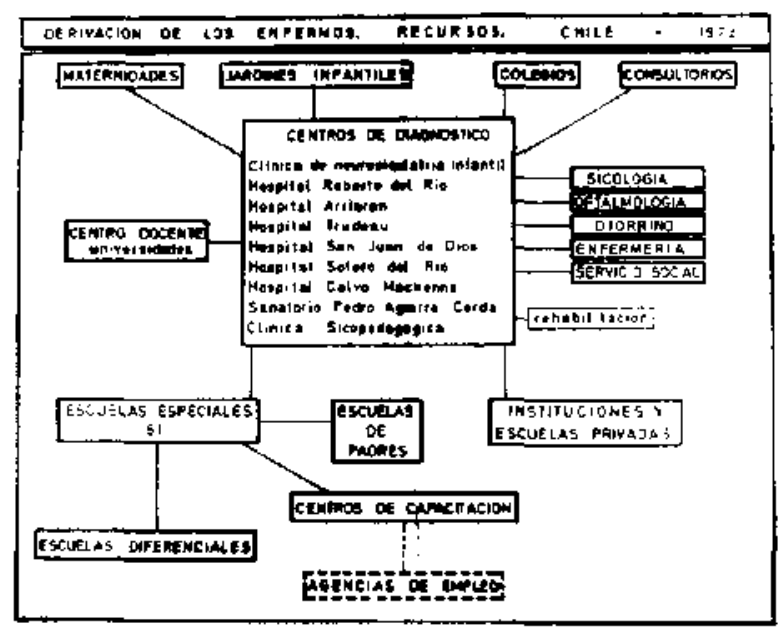

Figura N: 1. ren en su estimación para la población general y la diferencia entre ambos es de 20.000 afectados.

En el mismo cuadro se observa también que la DCM, sería dentro del grupo de enfermedades que estudiamos una patología de muy alta frecuencia.

En la tabla No 3 se pone de relieve la importancia cuantitativa del rubro de las alteraciones sensoperceptivas y destacamos el estrabismo y los problemas auditivos que pueden llegar a cerca de 
que son portadores los enfermos. En este momento al equipo de salud y al de educación les cabe un papel de la mayor importancia y responsabilidad.

El $2^{\circ}$ nivel lo constituyen los Centros de Diagnóstico, que son en su mayor parte los Servicios de Neurología y Psiquiatría de los Hospitales pe. diátricos. Estos Servicios trabajan en íntima conexión con aquellos que colaboran, ya sea en la pesquisa, diagnóstico, asistencia y tratamiento, o profilaxis de los cuadros que comentamos. (No se mencionan los Servicios de provincias), Las Universidades, por su parte forman a los profesionales que atenderán a los niños enferımos.

E1 3er. nivel lo forman los Centros de Ensenanza $\mathrm{y}$ rehabilitación. Así, las Escuelas Especiales, de las cuales hay 61 en Chile y son dependientes del Minjsterio de Educación, se abocan a la educación de los portadores de PC., RM., sensoperceptivos, etc. En conexión con ellas están las Escuelas de Padres y las Escuelas de Educación Diferencial, (15) que consisten en Escuelas para niños que no requieren educación especial. pero que su nivel de rendimiento escolar actual no es suficiente para la educación común. Alcanzando un nivel adecuado, el niño ingresa a la educación normal.

También existen las Escuelas Especiales privadas y las Instituciones que son estatales o particulares donde los niños reciben Asistencia y Tratamiento integral. Debemos decir también que en la mayoría de los Centros de Diagnóstico bay equipos de rehabilitadores físicos.

Un $4^{\circ}$ nivel logrado, son los Centros de Capacitación, cuyo papel los consideramos decisivo para el futuro desarrollo del niño. Funcionan con el propósito de. .. "formar una conducta con conocimientos y mecanismos habituacionales teóricos y prácticos que les permita a los alumnos egresar como hombres útiles e integrados a la sociedad común" (16).

En Chile, en este año 1972 se ha creado "e] primer Centro de Capacitación Laboral para niños impedidos y deficitarios" (16).

Por último, y a nivel teórico en nuestro país. mencionamos las "Agencias de Empleo", organismos públicos o privados destinados a dar trabajo a los diños que han dejado la escuela o el Centro de Capacitación (17) (18) (19).

Hasta aquí, la organización "espontánea" de los servicios que en Chile se ofrecen a los niños con Daño Neurológico. Decimos "espontánea" porque hasta el momento, no obedece a una estructura planificada.

Con respecto a las Escuclas Especiales, en la tabla No 4 se muestra el enorme déficit en la cobertura de atención. Se observa que alrededor del 95\% de los Deficientes Mentales, impedidos físicos e irregulares sociales quedan en nuestro país al
TAB L A Nọ 4

ESCUELAS ESPECIALES. MATRICULA, COBERTURA, CHILE, 1972

Matrícula Total 11.060

\begin{tabular}{lcc}
\hline & Cobertura & Déficit \\
\hline Deficientes mentales & $2.8 \%$ & $97,2 \%$ \\
Impedidos físicos & $2.3 \%$ & $97,7 \%$ \\
Irregulares sociales & $5.7 \%$ & $94,3 \%$ \\
\hline
\end{tabular}

margen de los beneficios que brindan aquellas Escuelas (12). De los 11.060 alumnos matriculados sólo 7.560 corresponđen a atención permanente. Además, sólo el $25 \%$ de los niños que siguen cursos en la Educación Especial son portadores déficits sicofísicos y el resto, representa a niños portadores de problemas socio-económicos.

Cuatro ideas principales podemos deducir de todo lo anterior:

1ㅇ- Que en Chile existe una estructura de formación "espontánea" para la atención de los tiños con Daño Neurológico.

$2^{\circ}$ - Que existe un abrumador déficit en lo que a Educrición Especial se refiere.

3\%- Que un porcentaje alto de los niños matriculados en las Escuelas Especiales no son portadores de daños neurológicos.

$4^{\circ}$ - Que comienza a funcionar en nuestro país la Capacitación Laboral para los niños impedidos, pero que aún no existen organizaciones que propendan a darles trabajo estable remunerado, competitivo o no.

Habríamos querido exponer ahora los recursos que se requerirán para mejorar la atención en este campo de la salud en nuestro país. Lamentablemente aun no existe un Programa de Salud Mental a nivel infantil en Chile. Por eso que anteriormente mencionábamos la estructura de atención espontánea. Consecuentemente, no podernos en este momento formular las necesidades de rccursos. Por otra parte el diagnóstico de la situación en el amplio campo de la salud mental infantil no ha sido formulado a nivel oficial. Sin embargo, los antecedentes sobre Magnitud del Problema, déficit de cobertura y de organizaciones que se hagan cargo del problema que hemos bosquejado sólo para algunos aspectos, permiten a nuestro juicio, prever que las necesidades de recursos son enormes en nuestro país.

No obstante to anterior podemos afirmar que en este momento sc está elaborando "en la Sec- 
ción Salud Mental del Servicio Nacional de Salud el Programa de Salud Mental Infanto-Juvenil, integrado al Programa Infantil, adultos, Atención Integral de la Mujer y demás Programas del Sector Salud y Multisectorial. Se plantea a través de un diagnóstico multifactorial en que los desórdenes mentales comprometen al $40-48,2 \%$ de los menores de 21 años. Se plantea la atención integral fundamentada en niveles de delegación de funciones multidisciplinarias; la profilaxis a nivel del ambiente y de las personas y enfatiza acerca de la atención ambulatoria, hospitales diurnos e incorporación efectiva de la comunidad a los trabajos que se propongan" (13).

Dentro de una perspectiva más amplia, diremos que en USA., por ejemplo: "Abrase cualquier plan estatal sobre retardo mental o Salud Mental y se describirá una enumeración cuidadosa de los muchos servicios disponibles"... (20). Sin embargo no siempre estas prestaciones actúan relacionadas entre sí, lo cual le resta eficacia al sistema. Por esta razón creemos que es muy importante tener claramente presente que los niños requieren "Asistencia" o sea la totalidad ininterrumpida de tratarnientos que enfocan al enfermo como un todo, como un ser somático, intelectual, emocional y social. El concepto de "tratamiento", sc refiere a las medidas destinadas a curar o mejorar un trastorno, una incapacidad, consideradas aisladamente (17).

En consideración a lo anterior pensamos que los Programas destinados a la Asistencia de estos niños deberían enfatizar sobre los siguientes aspectos.

1.- El papel del equipo médico debería ser además de sus funciones específica de diagnóstico y valoración integral, el de guiar y aliviar con vigilante atención tanto al enfermo como a su familia. (17) (20) (21). Sin embargo, no siempre el médico y su equipo tienen los conocimientos, experiencia y tiempo suficiente como para resolver los múltiples problemas, muchos de ellos extramédicos que plantean estos enfermos, (22) todo lo cual supone que los equipos de salud deberát tener adiestramiento térico y práctico con el fin de que los Programas tengan un rendimiento efectivo.

2.- Básicamente la Asistencia debe centrarse en la familia (21) y enfocarse hacia las necesidades del niño. Para estp, es preciso comenzar con la educación de los padres y trazar con ellos el plan a seguir, fundado en los requerimientos del pequeño y en los servicios disponibles para satisfacerlos (17).

Hay estudios que revelan cómo la presencia de un niño con PC. produce en un alto porcentaje desorganización del medio familiar. (20).
Otros trabajos han señalado que sólo el $52 \%$ de los padres están satisfechos acerca de cómo $\mathrm{cl}$ médico maneja el problema. (20). En fin, otros han señalado que la impresión sobre los progresos, logrados por niños en Asistencia, es pesimista, ya que la mayoría espera adelantos más efectivos que los observados. (5). Con estas citas sólo queremos insistir acerca de los complejos problemas que plantea la familia y sobre la responsabilidad que tiene el equipo médico para resolverlo.

3.- El período de Asistencia es largo, (17) como a largo plazo son los resultados. Por esta razón los programas deben ser orientados a atender las necesidades médicas normales, (crecimiento y desarrollo, socialización, juegos, inmunizaciones, etc.) y a las necesidades especiales (fisioterapia, alimentación, cirugia, etc.).

4. - La participación de la Comunidad en la programación y realización de los planes es fundamental. El niño está inserto en una familia y y ésta en una comunidad y de su interrelación dependerá gran parte del éxito. El médico por su parte debe estar preparado para integrarse a otros grupos multidisciplinarios. (17) (20).

Por último, quisiéramos insistir y hacer nuestros pensamientos de Yuliuns Cohen, (11) para quien el adiestramiento actual de los médicos en este campo, constituye una gran deformación, por cuanto en general, se exagera acerca de los aspectos puramente físicos (Hidrocefalia, convulsiones. trastornos genéticos, etc.) restando importancia a los factores socio-económicos, culturales y ambientales que rodean $\mathrm{y}$ a veces determinan la evolución de los cuadros.

\section{PROFILAXIS:}

Vamos a plantear la profilaxis de los Daños Neurológicos en el niño dentro de 2 ámbitos, cada uno con características bien determinadas. Creemos que así se clarifica el problema y se obtienen conclusiones de valor práctico.

Llamaremos profilaxis primaria al conjunto de medidas que debieran tomarse a fin de evitar hasta donde sea posible, la aparición en un niño de un daño neurológico.

Por profilaxis secundaria entenderemos al conjunto de medidas que debieran tomarse a fin dc evitar, que un daño dado se perpetúe o se profundice. Es decir, en este aspecto, se trata de intervenir directamente en la evolución natural de la enfermedad, haciendo posible la Asistencia y el Tratamiento.

Profilaxis Primaria: En la tabla No 5 (Pearson) se destacan los factores de más alto riesgo para producir daño. Dentro de ellos, queremos 
TABLA No S

FACTORES QUE AUMENTAN LL PELTGRO DE

RETARDO MENTAL (Y DE OTROS FACTORES NEUROLOGICOS) EN NINOS

\section{Factores maternos}

Edad menos de 16 an̆os o nayor de 40 años (más de 35 años para una primigrávida).

Las madres que entrañan mayor riesgo desde el punio de vista estadístico son las solteras, de talla escasa, delgadas, desnutridas, sin educación, de raza de color y pobres.

Antecedente de abortos, partos de mortinatos, muertes neonatales, niños vivos o mortinatos que pesaron menos de $2.000 \mathrm{~g}$. desprendimiento prematuro de ta placenta, placenta circunvalada.

Enfermedades y trastornos: hipertensión renal, proteinuria, nefritis, bacteriuria, diabetes sacarina, flebitis, en . fermedades tiroideas, hábitos de medicamentos, toxo. plasmosis, volumen cardíaco pequeño, retardo mental.

Tabaquismo excesivo.

Insuficiencia del cuello uterino.

Pelvis contrahecha.

Tumor cervical.

\section{Complicaciones de la gestación}

Hemorragia original en segundo o tercer trimestre.

Choque hemorágico.

Polihidramnios.

\section{Factores perinatales.}

Edad de gestación menor de 34 semanas.

Corren estadísticamente mayor riesgo los varones primo géritos y los gemelos prematuros.

Estado al nacer: puntuación Apgar baja, cianosis, necesidad de reanimación, presencia de insuficiencia respiratoria.

Hipoxia intrauterina concomitante con prolapso del cor. dón umbilical, desprendimiento prematuro de la placenta, toxemia gravídica o pelvis contrahecha.

Operación cesárea después de ensayo de parto.

Extracción con aplicación media de forceps o presentación de nalgas.

\section{Factores neonatales}

Necesidad de incubadora u oxígeno.

Succión, alimentación o llanto anormales.

Lesiones $y$ anomalías.

Asimetría de cara y extremidades.

Ictericia, tono insuficiente, convulsiones.

Aumento escaso de peso.

Vómitos y fiebre.

* Modificado de E. Denhoff: Cerebral Palsy: The Preschool Years, Springfield. III. Charles C. Thomas, 1967. recalcar los factores perinatales que según algunos autores como ya lo hemos visto son frecuentes $y$ sobre todo son los que con mayor facilidad podemos manejar. En este grupo, ya mencionamos la relación entre hipoxia y prematuridad con Danoo Neurológico (PC., RM., etc.). Deseamos dar otro argumento para enfatizar la importancia de estas 2 causales. En Chile (8) (9), también estos dos rubros son relevantes en la mortalidad perinatal, ya que ambos representan el $51 \%$ de las muertes perinatales con certificado de defunción dado por médico.

La prevención de los factores mencionados, implica la consideración de grandes capítulos de la Obstetricia y la Pediatría, es decir, el control del embarazo, la óptima atención del parto y los cuidados en el período de recién nacido.

Dentro de las lesiones prenatales, el enfoque de su profilaxis nos parece debe ser abordado teniendo claro el momento en que se producen las lesiones.

Para Lindemann (2) estas pueden ocurrir en las células germinales de los padres, son graves por que se traducen en factores letales, abortos o diversas malformaciones; por esto, evitar las intoxicaciones (Plomo, alcohol, nicotina, etc.) las radiaciones innecesarias y propender a mejorar las condiciones de vida de los padres, constituyen importantes elementos en la profilaxis.

Las "kicmopatías" (fruto del vientre) corresponden a las lesiones ocurridas desde la fecundación al nacimiento. Los 2 primeros estados, Blasto y Embrión son sensibles de manera importante a los gérmenes y los trastornos que ellos producen, no son germen-específico sino fase-especifico; por lo tanto llevan a la detención del proceso de organogénesis ocasionando diversos daños a los parénquimas según el momento en que se producen de acuerdo a lo señalado en el llamado Horario embriopático de Bourquin. (2).

Dentro de las embriopatías destacan la citomegalia, gripe y la embriopatía por excelencia que es la rubéola. Su prevención lógicamente es evitar la exposición al contagio de las embarazadas y la exposición de las jovencitas a la enfermedad. Pareciera más efectivo que la Gama globulina administrar a la embarazada $20 \mathrm{cc}$, al $2^{\circ}$ y 3 er. día después del contacto, de suero convalesciente. Algunos piensan que el aborto terapéutico no está indicado como profilaxis de una malformación, porque sólo puede contarse con ella en el $12 \%$ de los casos (2).

Desde un punto de vista diferente es importante mencionar el decisivo papel del pediatra en la profilaxis de los Daños Neurológicos mediante el Consejo Genético. (23) (24). Reconocemos que la cuestión no es fácil por la carencia de Centros destinados a este objeto, pero queremos destacar las enormes proyecciones que la genética 
puede brindar en la profilaxis de las cada vez nús numerosas enfermedades genéticas. Los exámenes de selección bioquímica permiten identificar a los afectados tempranamente y darles el tratamiento adecuado, así como ofrecerles orientación genética a los progenitores portadores de genes mutanics determinados. El uso de estudios meióticos es probable que permita identificar reorganizaciones cromosómicas de la índole de las inversiones, que no pueden reconocerse mediante las actuales tecnicas de estudio. La amniocentesis ha permitido diagnosticar algunos defectos genéticos in el feto. to cual posibilita luego de exámenes cromosomicos y bioquímicos, su tratamiento o modificación, como lo es en este momento la transfusión intrauterina para la eritroblastosis fetal grave.

En la actualidad, la orientación genética suele hacer que las personas tomen una decisión, por sí mismas como tenec más hijos o no, fundándose riesgos genéticos conocidos. Este problema involucra situaciones acerca del aborto terapéutico, inseminación artificial o la llamada ingenería genética (métodos que curarian o modificarían de manera eficaz genes mutantes o la expresión de los mismos) sobre las que pesan consideraciones religiosas, biológicas y legales.

Profilaxis Secundaria: El médico en este aspecto tiene la primera responsabilidad porque a menudo es el primer profesional que observa un desarrollo más lento, una reacción anormal o un defecto sico-físico cualquiera. (11). El deber reside en hacer el diagnóstico en forma precoz y correcta. Cuanto más grave el defecto, más temprano tiene la obligación de pesquisarlo. Esto no incluye que no deba esforzarse en detectar los casos más leves, que son los más frecuentes. No siempre sin embargo sucede así como lo comprueba un estudio hecho en un Condado de Nueva York en el que se demostró que muy pocos casos de retardos mentales habían sido informados entre pre-escolares, pero esta información experimentó un aumerto brusco en el $2^{\circ}$ y 3 er, año de la escuela.

A nadie escapará la importancia de un diagnóstico temprano. (11) (17) (18) (25). El abre grandes esperanzas de Asistencia y Tratamiento si pensamos que el potencial de desarrollo de un niño dañado neurológicamente, lo podemos moldear, hasta cierto punto, con numerosos procedimientos que a nuestra disposición se encuentran hoy día.

El descubrimiento temprano, excepto en los cuadros muy definidos exige del médico lo siguiente: (17).

1) Conocimientos firmes del desarrollo infantil.

2) Alto índice de sospecha cuando concurren en un niño los "factores de alto riesgo".

3) Control periódico en las etapas de lactante $y$ pre-escolar.
4) Prucbas sistemáticas de selección como fenilcetonuria.

5) Empleo sistemático de la Prueba de Denver (26) It otra similar. Esta prueba no brinda (1., ni cuociente dc desarrollo, pone en sobreilyiso al médico acerca de la posible existencia de un daño neurológico y lo hará emprender otros estudios si fuera necesario.

Es necesacio mencionar el aporte que han significado las experiencias hechas en pre-escolares en USA., (Head Start Program) que han comprobado la "eficacia de la estimulación y el enriquecimiento temprano para dismisuir el choque am. biental en la realización de los niños" (11).

Por otra parte, pensamos que semejante criterio profiláctico deberá ser usado en los casos del llamado sindrome del niño maltratado y en los muy numerosos casos de deprivación socio-cultural. (4) $(27)$.

\section{5. ¿QUE SE PUEDE ESPERAR DE ESTOS NINOS?}

Cuando nos planteamos ¿qué podemos esperar de los niños con Daño Neurológico?, nos parece importante clarificar previamente algunas cuestiones. Desde luego, tenemos que especificar, cuál es nivel óptimo que esperamos en determinado niño, cuál es su diagnóstico, incluyendo en él, su capacidad intelectual, el defecto físico concomitante y su nivel de educación actual. Además tendremos que considerar cómo es su ambiente socioeconómico y farniliar (5) (28) y por último cuáles son nuestras reales posibilidades para brindarle asistencia y tratamiento

En términos muy generales desearíamos para todos, niveles adecuados de productividad económica personal, obviamente es ésta una posición teórica, pero si logramos por lo menos que los más incapacitados obtengan una actividad de vida diaria (AVD) independiente habrán dejado de ser una carga para la familia, por lo menos en el sentido social y esto significa evidentemente economizar cuidados especiales, que significan di. nero.

Lo anterior, es una situación muy frecuente en $\mathrm{PC}$ en la que la marcha se considera un nivel muy alto de éxito. Este logro está en íntima relación con numerosas variables (defectos concomitantes $\mathbf{u}$ asociados, posibilidades quirúrgicas, topografía de la lesión, etc.), siendo una de las más importantes el CI (2).

Las posibilidades de atención integral como lo hace el IRI, (Instituto de Rehabilitación Infantil) también inciden en obtener buenos resultados y como vernos, (figura No 2) un alto número de niños ingresan al Instituto sin poder caminar y 


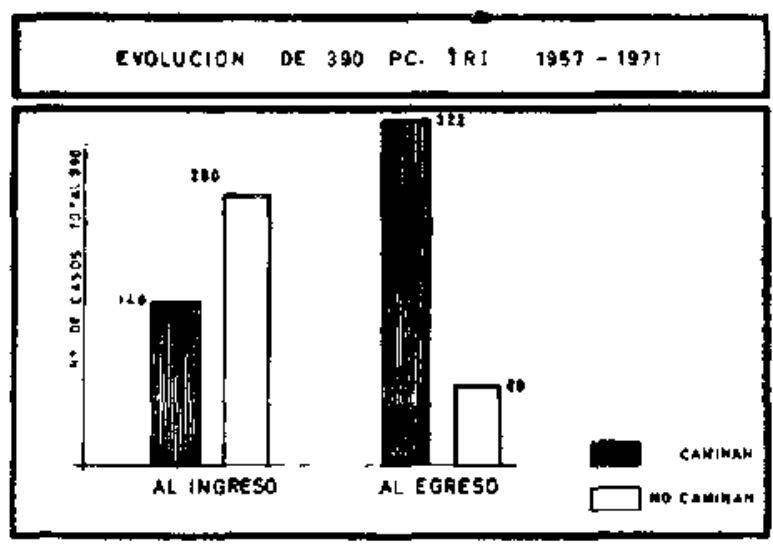

Figura Nọ 2.

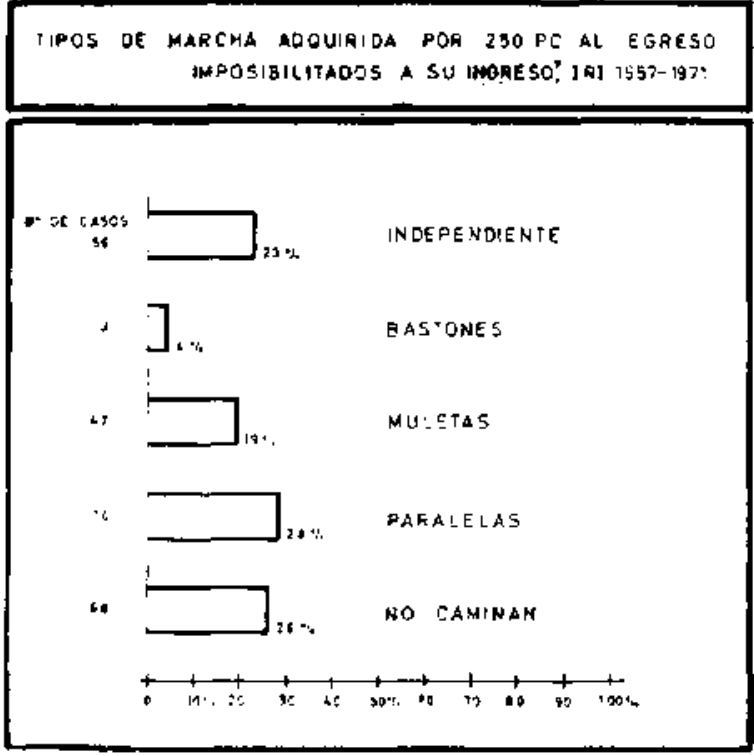

Figura No 3.

egresan de él con algün tipo de matcha, (figura No 3 ).

Sin embargo, no debemos dejarnos seducir por estos resultados, ya que si tomamos globalmente los 7 recién nacidos por 100.000 que nacen con $P C$ veremos, que sólo en 3 de ellos podremos actuar con alguna esperanza de éxito (2).

Con respecto a los prematuros los datos son contradictorios. Un estudio (28) señala que el $50 \%$ serán ineducables en escuelas normales, el $20 \%$ requerirán tratamiento especial en escuela normal y sólo el $25 \%$ tendrá un CI de distribución normal. Lindeman (2) por su parte señala que el $85-90 \%$ de los prematuros que sobrevivan serán normales. Tan notables diferencias podrán deberse a características de la muestra, oportunidad de tratamiento, grado de maduración, etc.

Enfocando el panorama bacia el ámbito más especializado de las enfermedades metabólicas, podemos decit que algunas tienen tratamiento y quc en otras, Fenilcetomeria, por ejemplo, su éxito depende de la precocidad con que se instale la terapia. (29).

Desde el punto de vista de la educación y de lu cylue de clla podemos esperat en los RM, O. Johson (21) da una clasificación que nos parece

TABLA No

NIVELES EDUCACIONALES EN RETARDADOS MENTALES (Johnson 1968)

1 - R.M. GRAVE $0,5 \%$

2 - R.M. ADIESTRABLE $<\begin{aligned} & \text { Empleo no competitivo } \\ & \text { Se cuidan por sí mismos }\end{aligned}$

3 - R.M. EDUCABLE Directamente empleables $3 \%$ Empleo diferido

4 - SUJETOS LENTOS PARA APRENDER $17-18 \%$

operante, (tabla No 6). Del RM. grave nada podemos esperar en cuanto a sí mismo ni en cuanto a productividad, porque necesitarán asistencia guardiana de por vida. El RM adiestrable poede alcanzar una semi independencia social y en un ambiente vigilado es capaz de aportar algo en su casa o en un taller protegido o en una institución. Para ellos y dicho sea de paso el mejor enfoque terapéutico es el método del condicionamiento operante, (21) (27). Los RM. educables, ya más frecuentes que los anteriores, tienen patencialidades de independencia social y económica y pueden ganarse la vida como obreros calificados o no y para ellos los programas educativos no serán muy diferentes de los que reciben los niños normales. Este grupo como se ve en el cuadro tiene dos categorias en cuanto a posibilidades de empleo.

Con respecto a la epilepsia, ella plantea problemas del mayor interés. Debemos considerar que el CI no influye significativamente en estos enfermos puesto que su distribución es similar al de Ia población general. (Según Lennox el 5\% de los epilépticos su CI es menor que 71 y en el $18 \%$ mayor que 121) (19). Por otra parte desde el punto de vista de la crisis, el $80 \%$ podría tener un empleo remunerado sin peligros de aparición de ataques, levando un buen tratamiento.

Se estima sin embargo que el $60 \%$ de los patrones de USA no emplean a un epiléptico por ningún motivo, aduciendo razones de mayor frecuencia de accidentes y el consiguiente pago de indemnizaciones. En realidad, estudios efectuados, han demostrado que un epiléptico es capaz de cualquier trabajo. Otros estudios han demos- 
trado que a cllos, se les otorga en general menos indemnizaciones que a los diabéticos, cardiópatas o artríticos (19).

Ante esta situación de prejuicios infundados (25), se están dictando cn USA disposiciones legales a fin de modificar el trato discriminativo para los epilépticos (19) (23).

Con respecto a la DCM, estos niños pueden injustificadamente ser relegados a educación especial, o caer en excesos sociales si su problema no es enfocado en forma correcta (30).

\section{Bibliografía}

1.-Denhoff, E. ana Pick, 1.: Cerebral Palsy and related disorders. New York. Mc. Graw-Hill, 1960.

2.-Linderman, $\mathcal{K}_{\text {.: }}$ La Parálisis Cerebral. Barcelona. Científico-Médica, 1968.

3.- Paine, R.: Sindromes de "Lesión Cerebral Mínima". Clín. Peđiát., 15: 779, 1968.

4.-Meneghello, J.: Pediatría. Buenos Aires, Inter-Médica, 1972.

5.-Ancelovici, S.: Parálisis Cerebral Infantil. Contribución a su estudio. Tesis de títtio. Universidad de Chile, 1970.

6.-Fliman, M.: Un diagnóstico de Ja situación de la Salud Mental en Chile. Rev. Méd. Chile. 100: 343, 1972 .

7.-Power, H.: Problemas de Salud Mental de la edad escolar en el gran Santiago. Santiago-Chile, Sub Departamento Fomento de la Salud, Servicio Nucional de Salud, 1966.

8.- Servicio Nacional de Salud. Defunciones y causas de muerte. Anuario 1971, Chile, 1972.

9.-Servicio Nacional de Salud. Nacimientos. Anuario 1971, Chile, 1972.

10.-Meneghello, J., Rosselot, J. y Manterola, A.: Lecciones de Pediatría Clínica y Social. SantiagoChile. Edición Universitaria, 1964.

11.-Cohen, J.: Rehabilitación Vocacional del retardado mental. Clín. Pediát., 15: 1017, 1968.

12.-Ford, F.: Enfermedades del Sistema Nervioso. Rosario (Rep. Argentina). La Médica, 1967.

13.-Departamento Satrd Memtl. Apuntes S.N.S., 1972.

14.-Bravo, L. Trastornos del desarrollo psicológico en niños con daño cerebral mínimo. Rev. Chil. Ped. 39: $369,1968$.

15.--Documemos para organización de cursos diferenciales. Ministerio de Educación. Sección Educ. Ex+ perimental. Santiago, Chile, 1972.
16.-Editorial. El niño limitado. 4: 97, 1972.

17.- Pearson, P.: Papel del Médico en el diagnóstico y la asistencia de retardados mentales. Clín. Pediát. 15: 835,1968 .

18.- Walli, J. $E, W$. El niño deficiente físico, mental $y$ emocional, Buenos Aires, Paidós, 1965.

19.- Cronther, D. L.: Aspectos Psicosociales de la Epilepsia. Clín. Pediát. 14: 921, 1967.

20.-Fremont, A. C.: Uso de los Servicios de la Comu. nidad: envio a especialistas y consulta. Clín. Pediát. 15: $989,1968$.

21.-Joimson, $O$. G.: Educación especial para el Retardo Mental. Clín. Pediát. 15: 1005, 1968.

22.-Zuckerberg, H. D. y Snow, G. R.: ¿Qué esperan los padres del médico? Resumen de opiniones recientes. Clín. Pediát. 15: 871, 1968.

23.- Taylor, D. C. and Bower, B. O.: Prevention in Epileptic disorders. Lancet, 1971, 1136.

24.-Wright, S, W. and Sparkes, R. S.: Orientación Genética en el Retardo Mental. Clín. Pediát. 15: 905, 1968.

25.-Mc. Connet $F_{i}$ A new approach to the Management of Chilhood deafness. Ped. Clín. 17: 397, 1970.

26.-Fratkenburg, W. K. and Dodds, Y. B.: The Denver Developmental Screening Test. J. Pediát. 7: 181 1967.

27. - Bijow, S.: Modificación de la conducta en retardados mentales. Clín. Pediát. 15: 969, 1968.

28,-Drilfiem, $M_{.:}$Incidencia de Trastornos Mentales y Físicos en escolares de muy bajo peso al nacer. Pediatrics, Marzo 1961.

29.-O'Fiynn, M. E.: Trastornos mentales concomitantes con retardo mental. Clin. Pediát. 15: 889, 1969

30.-. Clements, S.; National Society for Crippled Children and Adults. Chicago, 1964.

Este trabajo es una recopilación de apuntes correspondientes a la clase dictada por los autores en el III. Scminario Latinoamericano de Salud Pública MaternoInfantil efectuado en Noviembre de 1972, por el Departamento de Salud Pública y Medicina Social de la Universidad de Chile.

Los autores agradecen a la Dirección y Personal del Instituto de Rehabilitación Infantil de Santiago de Chile, (IRI) las facilidades otorgadas para la realización de este estudio. 